\section{PERFIL EPIDEMIOLÓGICO DOS ACIDENTADOS E FATORES AMBIENTAIS QUE FAVORECEM ACIDENTES OFÍDICOS BOTRÓPICOS, NO ESTADO DO TOCANTINS, BRASIL}

\author{
Epidemiological profile of the injured and environmental factors that favor botropic \\ snake accidents, in the State of Tocantins, Brazil
}

Perfil epidemiológico de los factores lesionados y ambientales que favorecen los accidentes de serpientes botrópicas, en el Estado de Tocantins, Brasil

\section{Desafios}

Artigo Original

Original Article Artículo Original

\author{
Deize Carneiro Queirós ${ }^{1}$, Antônio Italo dos Santos Nunes ${ }^{2}$, Gessi Carvalho de Araújo \\ Santos $^{3}$, Raiany Cristine Cruz da Silva ${ }^{1}$, Carla Simone Seibert ${ }^{* 1,2}$ \\ ${ }^{1}$ Programa de Pós-Graduação em Ciências do Ambiente, Universidade Federal do Tocantins, Palmas - TO. \\ ${ }^{2}$ Curso de Ciências Biológicas, Universidade Federal do Tocantins, Porto Nacional - TO. \\ ${ }^{3}$ Programa de Pós-Graduação em Ciências da Saúde, Universidade Federal do Tocantins, Palmas - TO.
}

*Correspondência: Curso de Ciências Biológicas, Universidade Federal do Tocantins, Rua 03, Qd. 17, S/N, Jardim dos Ipês, Porto Nacional, Tocantins, Brasil.CEP:77.500-000.e-mail seibertcs@uft.edu.br

Artigo recebido em 06/04/2020 aprovado em 04/11/2020 publicado em 04/03/2021.

\title{
RESUMO
}

O objetivo desse trabalho foi avaliar o perfil epidemiológico dos acidentados por serpentes botrópicas e os fatores ambientais que facilitam os encontros dos humanos com essas serpentes, que são popularmente conhecidas como jararacas. O perfil epidemiológico foi realizado para subsidiar o entendimento da dinâmica desse agravo no Estado do Tocantins, com dados de 2007 a 2019, obtidos da plataforma TabWin-SINAN. Para correlacionar os fatores ambientais com os acidentes botrópicos foram obtidos dados no site do IBGE e no INMET, para as cidades de maior notificação: Araguaína, Palmas e Porto Nacional. Nesse período, foram registrados 7.906 acidentes botrópico, com predomínio do sexo masculino (76,7\%), na faixa etária de 20-59 anos $(62,0 \%)$. A maioria dos acidentes foi classificado como leve e moderado (89,6\%), com ocorrência de $0,4 \%$ de óbitos. As médias acumuladas mensais das variáveis ambientais (temperatura do ar, umidade relativa do ar e precipitação pluviométrica) foram fatores determinantes para os acidentes, com correlações significativas. Contudo, não foi observada correlação para o avanço agrícola. Portanto, os acidentes botrópicos no estado do Tocantins atingem a faixa etária mais ativa da população, sendo a pluviometria, temperatura do ar e umidade relativa do ar fatores importantes para o monitoramento desses acidentes, com relevância sazonal.

Palavras-chave: Acidente botrópico; Fatores ambientais; Epidemiologia.

\section{ABSTRACT}

The objective of this work was to evaluate the epidemiological profile of those injured by botropic snakes and the environmental factors that facilitate human encounters with these snakes, which are popularly known as jararacas. The epidemiological profile was carried out to support the understanding of the dynamics of this condition in the State of Tocantins, with data from 2007 to 2019, obtained from the TabWin-SINAN platform. In order to correlate environmental factors with botropic accidents, data were obtained from the IBGE website and INMET, for the cities with the highest notification: Araguaína, Palmas and Porto Nacional. During this period, 7,906 botropic accidents were recorded, with a predominance of males (76.7\%), aged 20-59 years (62.0\%). Most accidents were classified as mild and moderate (89.6\%), with $0.4 \%$ of deaths occurring. The monthly accumulated averages of environmental variables (air temperature, relative humidity and rainfall) were determining factors for accidents, with significant correlations. However, no correlation was observed for agricultural progress. Therefore, botropic accidents in the state of Tocantins affect the most active age group of the population, with rainfall, air temperature, and relative humidity being essencial factors for monitoring these accidents, with seasonal relevance.

Keywords: Botropic accident; Environmental factors; Epidemiology. 


\section{RESUMEN}

El objetivo de este trabajo fue evaluar el perfil epidemiológico de los heridos por serpientes botánicas y los factores ambientales que facilitan los encuentros humanos con estas serpientes, conocidas popularmente como jararacas. El perfil epidemiológico se realizó para apoyar la comprensión de la dinámica de esta condición en el estado de Tocantins, con datos de 2007 a 2019, obtenidos de la plataforma TabWin-SINAN. Con el fin de correlacionar los factores ambientales con los accidentes botánicos, se obtuvieron datos del sitio web del IBGE y del INMET, para las ciudades con mayor notificación: Araguaína, Palmas y Porto Nacional. Durante este período, se registraron 7,906 accidentes botánicos, con predominio de varones (76.7\%), con edades entre 20 y 59 años (62.0\%). La mayoría de los accidentes se clasificaron como leves y moderados $(89,6 \%)$, con un $0,4 \%$ de muertes. Los promedios acumulados mensualmente de variables ambientales (temperatura del aire, humedad relativa y lluvia) fueron factores determinantes para accidentes, con correlaciones significativas. Sin embargo, no se observó correlación para el progreso agrícola. Por lo tanto, los accidentes botánicos en el estado de Tocantins afectan al grupo de edad más activo de la población, siendo la lluvia, la temperatura del aire y la humedad relativa los factores importantes para monitorear estos accidentes, con relevancia estacional.

Palabras clave: Accidente botropico; Factores ambientales; Epidemiología

\section{INTRODUÇÃO}

Acidentes causados por animais peçonhentos provocam mortalidade, intoxicação e consequências graves em todo o mundo. O Brasil é o país com maior incidência de acidente ofídico na América do Sul, com 29 mil acidentes ofídicos por ano (Alcântara et al., 2018; SINAM, 2020). O país possui uma riquíssima diversidade faunística de serpentes, pois os trópicos apresentam condições ambientais favoráveis à adaptação e sobrevivência desses animais (Nogueira et al., 2019). O envenenamento causado por serpentes do gênero Bothrops, no Brasil, representa cerca de $90 \%$ dos acidentes e é mais frequente nas regiões Norte e Centro-Oeste do país (Pinho et al., 2008; Lima et al., 2009; Santos et al., 2014; Bernarde et al., 2015; Leobas et al., 2016; Parise, 2016; Ximenes et al., 2018).

O Estado do Tocantins está entre os nove estados que compõe a Amazônia Legal, cujo clima é predominantemente tropical seco e semiúmido, com uma estação seca e outra chuvosa bem definidas (Cavalcanti et al., 2012). A estação seca ocorre de maio a setembro, já o período chuvoso inicia no mês de outubro e termina em abril. É abundante em luminosidade e ideal para atender às necessidades do ciclo produtivo de grãos (Bridgewater et al., 2004; Brasil, 2011). Suas principais atividades econômicas são a agricultura e a pecuária, pois o Estado possui fitofisionomia que favorece o processo de mecanização agrícola. Nos últimos dez anos, a produção de grãos no Estado obteve um crescimento superior a $180 \%$ na área plantada e $240 \%$ na produção agrícola (Seagro, 2018).

Há um crescimento do acidente ofídico no Estado, e isso pode ter relação com o aumento do desmatamento e com o desenvolvimento das áreas de plantio, sendo necessário entender os fatores determinantes desses acidentes. Além disso, pouco se conhece sobre a biologia das serpentes do Cerrado tocantinense, sua relação com ambiente e a ocorrência desses acidentes, sendo que essas variáveis podem estar relacionadas aos acidentes ofídicos e às suas complicações (Lima et al., 2009; Silva, 2017; Alcântara et al., 2018). Portanto, o objetivo desse trabalho foi avaliar o perfil epidemiológico do acidentado por serpentes do gênero botrópico e os fatores ambientais que facilitam o encontro dos humanos com essas serpentes. 


\section{MATERIAIS E MÉTODOS}

\section{Perfil epidemiológico do acidente botrópico no} Tocantins

Essa etapa representou um estudo epidemiológico, retrospectivo, de abordagem quantitativa. Foram avaliados dados referentes aos acidentes botrópicos, no período de 2007 a 2019, por meio do TabWin, software utilizado para tabulação dos dados do Sistema de Informação de Agravos de Notificação (SINAN). Foram analisadas as variáveis epidemiológicas referentes ao indivíduo acidentado (sexo e faixa etária), ao atendimento hospitalar (gravidade e evolução do caso), ao local de ocorrência (município) e ao mês do acontecimento. Os dados foram tabulados em planilhas elaboradas no programa Excel® do Windows versão 10.

Precipitação pluviométrica, temperatura do ar, umidade relativa do ar e avanço agrícola com o acidente botrópico

Foi analisada a correlação entre as variáveis ambientais (precipitação pluviométrica, temperatura do ar e umidade relativa do ar) com o índice de acidente botrópico para os três municípios com maior registro de acidente botrópico (Araguaína, Palmas e Porto Nacional). Os dados foram obtidos a partir do Banco de Dados Meteorológicos do Instituto Nacional de Meteorologia (INMET), de cada município, para os anos de 2007 a 2019, analisados mensalmente e anualmente.

Para avaliar e fundamentar a análise do avanço agrícola no Tocantins, foram obtidas as informações no banco de dados do SIDRA, provenientes do Censo Agropecuário, para os anos de 2007 a 2018, com as varáveis de área plantada, produto da lavoura de grãos de feijão, milho, soja e mandioca, disponíveis no site do IBGE. Estes dados são informados por ano de atividade, portanto essas variáveis foram analisadas anualmente, por município (Araguaína, Palmas e Porto Nacional)

Para a análise estatística foi utilizado o teste de correlação de Pearson, com o software GraphPad Prisma (versão 5.03). As variáveis ambientais e do avanço agrícola foram as unidades de análise e a variável desfecho foi o número de acidente botrópico. Os dados foram coletados em abril de 2020.

\section{RESULTADOS E DISCUSSÃO}

O acidente ofídico é considerado um problema de saúde pública, devido ao elevado índice e suas consequências às vítimas, e por causar impactos sociais e econômicos significativos ao país. Contudo, nosso estudo focou no acidente botrópico por ser o responsável pela maioria dos acidentes do Tocantins. No período de 2007 a 2019, o acidente botrópico no Estado foi responsável por 7.906 acidentes e apresentou um padrão epidemiológico clássico. A maior incidência ocorreu em pessoas do sexo 65 masculino, com 6.125 acidentes (76,7\%), com distribuição heterogênea em relação à faixa etária dos acidentados. A população economicamente ativa (20 a 59 anos) foi a mais acometida (62\% dos acidentes). Crianças e adolescentes representaram 27,3\% dos acidentes e os idosos 10,7\% (Fig. 1).

Figura 1. Número de acidentes causados pelo gênero Bothrops, segundo faixa etária e sexo, no Estado do Tocantins, de 2007 a 2019. Fonte: SINAN (2020).

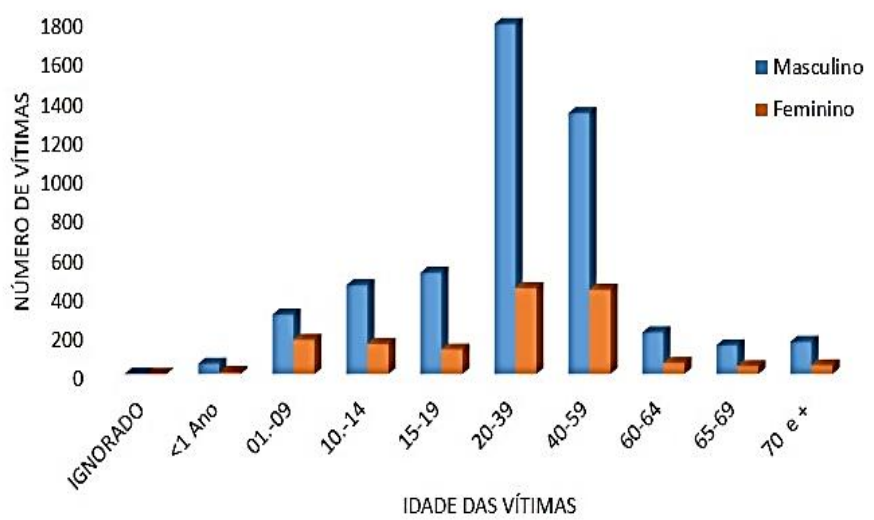


Dos acidentes registrados no período, $42,2 \%$ foram classificados como grau leve, 41,2\% como moderado, 5,0\% como grave, e 3,7\% ignorado/branco. Os casos que evoluíram para óbito representaram $0,4 \%$ dos acidentes (33), com a maior incidência nos graves, mas também nos moderados e leves (Fig. 2). Este é um número significativo tendo em vista a eficácia da terapia com o antiveneno, o que precisa ser compreendido a fim de buscar propostas para novas terapias coadjuvantes ao antiveneno e/ou ao manejo clínico dos pacientes.

Figura 2. Classificação da gravidade e letalidade dos acidentes causados pelo gênero Bothrops, no Estado do Tocantins, de 2007 a 2019. Fonte: SINAN (2020).

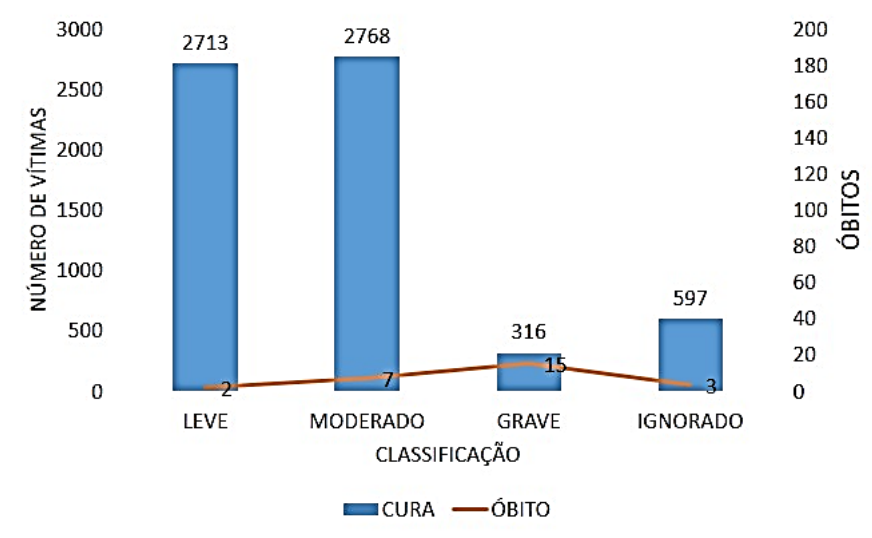

Nossos resultados corroboram estudos epidemiológicos realizados em todo o Brasil (Bochner e Struchiner, 2003; Pinho et al., 2008; Lima, 2009; Bernarde, 2014; Bernarde et al., 2015; Santana e Suchara, 2015; Ximenes et al., 2018). Bochner e Struchiner (2003) avaliaram o padrão epidemiológico dos acidentes ofídicos, em 100 anos, e concluíram que estes se mantiveram inalterados. Esse padrão retrata a predominância dos acidentes para o gênero Bothrops, sendo o trabalhador rural do sexo masculino o mais acometido, atingido principalmente nos membros inferiores. Os autores, por sua vez, identificaram a necessidade de estudos para avaliar a relação dos acidentes ofídicos com as variáveis ambientais e com os hábitos desses animais.
O registro de acidentes com serpentes do gênero Bothrops foi expressivo ao longo dos anos no Estado do Tocantins. O município de Araguaína apresentou o maior número de acidentes nesse período (Fig. 3), com total de 513 acidentes (6,5\%), seguido pela capital Palmas (329 acidentes; 4,2\%), e do município de Porto Nacional (272 acidentes; 3,4\%) (Fig. 3).

Figura 3: Distribuição dos municípios do Estado do Tocantins com os maiores números dos acidentes ofídicos acometidos pelo gênero Bothrops, de 2007 a 2019. Fonte: SINAN (2020).

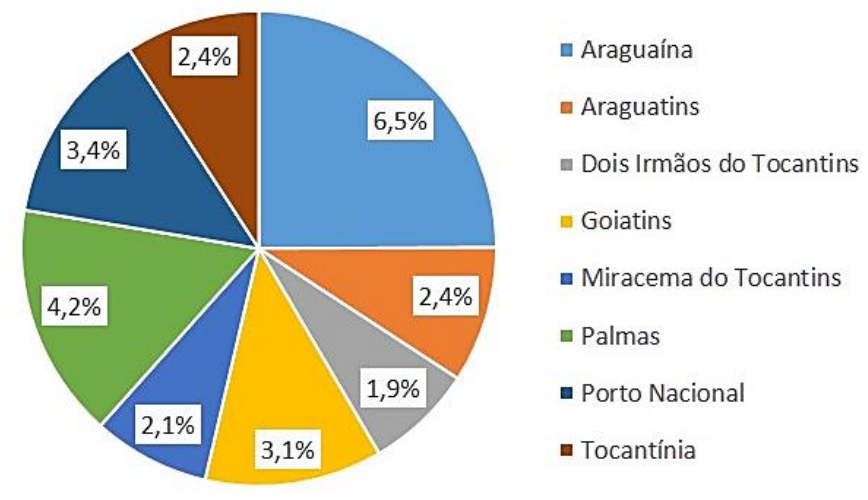

A correlação da temperatura do ar, umidade relativa do ar, precipitação pluviométrica e avanço agrícola com o acidente botrópico foi analisada para os municípios que apresentaram o maior número de acidentes (Araguaína, Palmas e Porto Nacional). A distribuição dos acidentes botrópicos, por mês de ocorrência, nessas cidades está apresentada na tabela 1 .

Houve aumento no número de acidentes no período de janeiro a maio, e redução de junho a setembro. A maior incidência ocorreu no mês de abril, com total de 129 acidentes registrados nas três cidades, seguido do mês de dezembro, com total de 122 acidentes. A menor incidência foi no mês de agosto, com total de 50 acidentes, seguido do mês de setembro (55 acidentes).

As maiores precipitações pluviométricas, nos municípios de Araguaína, Palmas e Porto Nacional ocorreram nos meses de janeiro a abril, e de outubro a 
dezembro. O mês de maior precipitação foi fevereiro $(17,0 \%)$, e a menor precipitação foi em agosto $(0,2 \%)$.

Para o acidente botrópico foram registrados os maiores percentuais no mês de abril (11,6\%), enquanto o mês de menor acometimento foi agosto (4,5\%), que coincidiu com o mês de menor índice pluviométrico. Foi observado aproximação entre os meses com maior precipitação pluviométrica e maior número de acidentes, com correlação significativa para os três municípios ( $\mathrm{r}=0,67 ; \mathrm{P}=0,01)$ (Fig. 4). Também houve correlação significativa quando as médias acumuladas mensais da umidade relativa do ar e da temperatura foram associadas (Araguaína $\mathrm{r}=0,73 ; \mathrm{P}=0,007$; Porto Nacional $r=0,76 ; P=0,004$; Palmas $r=0,83 ; P=0,008$ ) (Fig. 5).

Tabela 1: Acidente botrópico segundo o mês de ocorrência nas cidades de Araguaína, Palmas e Porto Nacional, de 2007 a 2019.

\begin{tabular}{lccc}
\hline $\begin{array}{l}\text { Mês / } \\
\text { Cidade }\end{array}$ & Araguaína & Palmas & $\begin{array}{c}\text { Porto } \\
\text { Nacional }\end{array}$ \\
\hline Janeiro & 53 & 32 & 30 \\
Fevereiro & 43 & 30 & 16 \\
Março & 46 & 30 & 28 \\
Abril & 49 & 41 & 39 \\
Maio & 49 & 35 & 27 \\
Junho & 42 & 20 & 21 \\
Julho & 39 & 24 & 13 \\
Agosto & 24 & 15 & 11 \\
Setembro & 27 & 17 & 11 \\
Outubro & 41 & 27 & 19 \\
Novembro & 35 & 29 & 29 \\
Dezembro & 65 & 29 & 28 \\
\hline TOTAL & 513 & 329 & 272 \\
\hline Fonte: SINAN & $2020)$ & & \\
\hline
\end{tabular}

Fonte: SINAN (2020).

Palmas, capital do Tocantins, possui importantes taxas de crescimento demográfico, e é o município mais populoso do estado (228.085 habitantes) (IBGE, 2015), está entre as capitais com as temperaturas mais elevadas do Brasil, alcançando temperaturas acima de $40^{\circ} \mathrm{C}$. O setor de serviços é o principal da economia palmense, com $57,5 \%$, e a agropecuária com $34,6 \%$ de participação. Araguaína é segundo município mais populoso (146.219 habitantes), destaca-se na criação de gado para cria, recria, engorda e abate, na produção leiteira e de produtos agrícolas. De 2007 a 2019, obteve um crescimento agrícola, passando de 2.832 he para 8.656 he de área plantada ou destinada à colheita. E o terceiro município com maior registro de acidente ofídico foi Porto Nacional, notável pelo potencial agropecuário, pois em 2016 obteve um crescimento de $293 \%$ de área plantada, em relação a 2007 (IBGE, 2019).

Figura 4: Distribuição mensal dos acidentes botrópicos e precipitação pluviométrica (média acumulada mensal) nas cidades de Araguaína, Palmas e Porto Nacional, do Estado do Tocantins, de 2007 a 2019. Fonte: SINAM/INMET (2020).

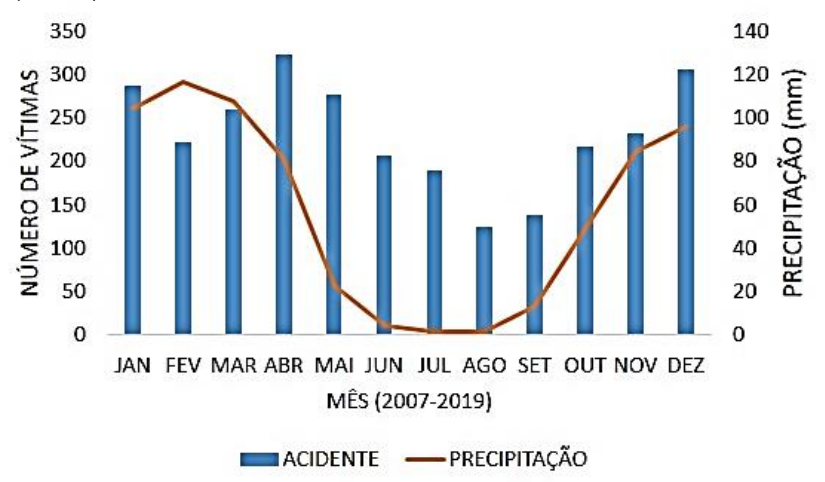

Por outro lado, não houve correlação significativa das médias acumuladas anuais para as variáveis ambientais e o avanço agrícola com o acidente botrópico (Tab. 2). O maior número de acidentes botrópicos para o município de Araguaína, pode estar relacionado com o seu clima, que é influenciado pela vegetação da Floresta Amazônica. De acordo com os resultados obtidos neste estudo (Fig. 5), o município tem temperaturas mais amenas quando comparadas com as temperaturas de Palmas e de Porto Nacional, o que pode favorecer o acidente, uma vez que, as serpentes encontram um habitat mais favorável para seu ciclo de vida. Outro fator é que o município também é referência em saúde na região norte do Tocantins, e dos municípios mais próximos dos estados vizinhos. Por esse motivo, pacientes vítimas de acidentes por animais peçonhentos são 
Figura 5: Média acumulada mensal da temperatura e umidade relativa do ar com o acidente botrópico, nos municípios de Araguaína, Palmas e Porto Nacional, de 2007 a 2019. Fonte: SINAM/INMET (2020).
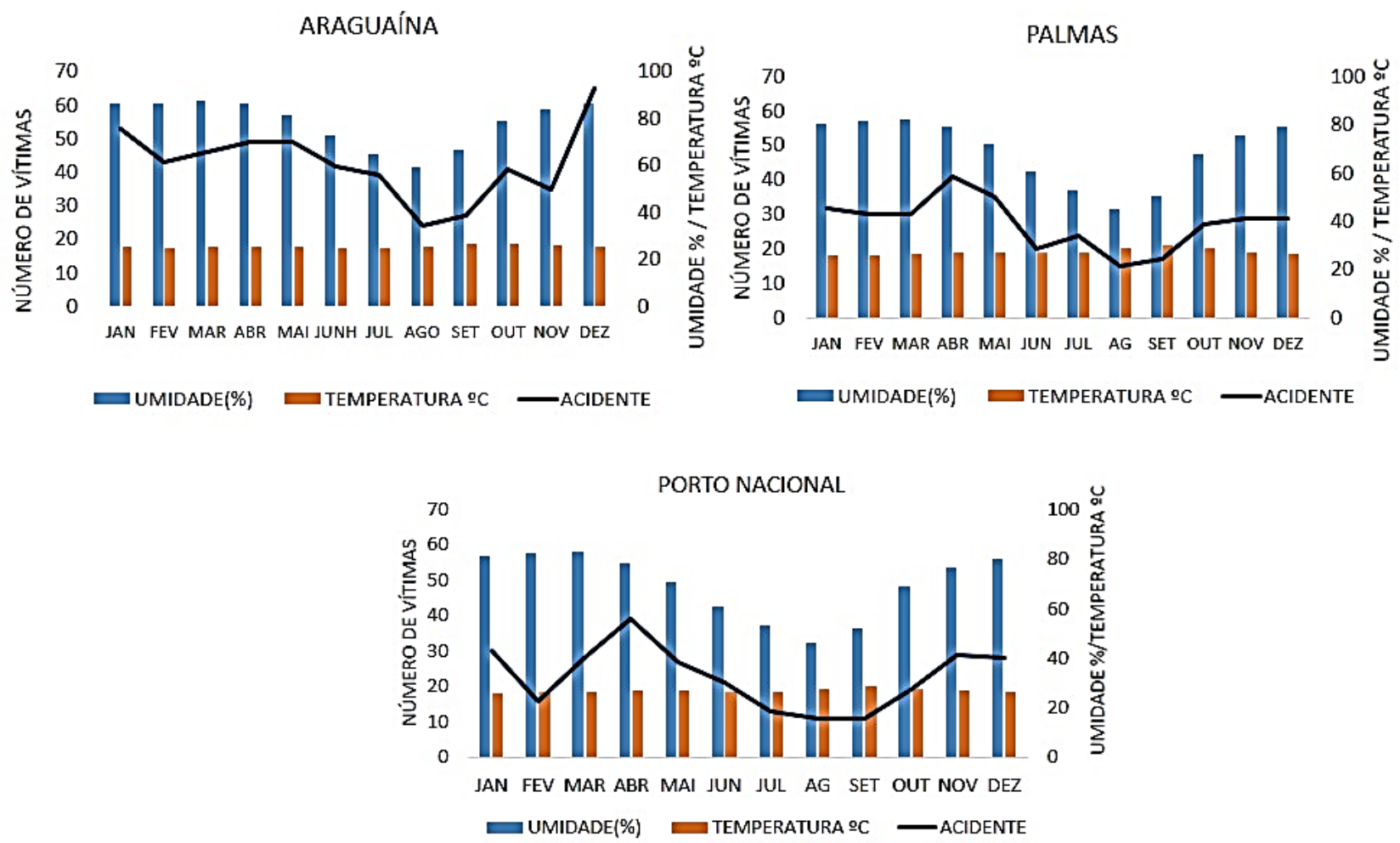

Tabela 2. Resultado da análise de correlação de Pearson para os resultados anuais de incidência de acidente botrópico segundo variáveis climáticas e o avanço agrícola, para os municípios de Araguaína, Palmas e Porto Nacional Tocantins, de 2007 a 2018.

\begin{tabular}{|c|c|c|c|}
\hline Variáveis & Araguaína & Palmas & P. Nacional \\
\hline \multirow[t]{2}{*}{ Temperatura } & $\mathrm{r}=0,11$ & $r=-0,09$ & $* \mathrm{ND}$ \\
\hline & $\mathrm{P}=0,72$ & $\mathrm{P}=0,77$ & \\
\hline \multirow[t]{2}{*}{ Precipitação } & $r=-0,09$ & $r=-0,42$ & $\mathrm{r}=0,12$ \\
\hline & $\mathrm{P}=0,78$ & $\mathrm{P}=0,15$ & $\mathrm{P}=0,71$ \\
\hline \multirow[t]{2}{*}{ Área plantada } & $\mathrm{r}=-0,18$ & $r=-0,08$ & $\mathrm{r}=0,06$ \\
\hline & $\mathrm{P}=0,58$ & $\mathrm{P}=0,79$ & $\mathrm{P}=0,85$ \\
\hline \multirow[t]{2}{*}{ Feijão } & $\mathrm{r}=0,54$ & $\mathrm{r}=0,45$ & $\mathrm{ND}^{*}$ \\
\hline & $\mathrm{P}=0,07$ & $\mathrm{P}=0,14$ & \\
\hline \multirow[t]{2}{*}{ Mandioca } & $\mathrm{r}=0,30$ & $\mathrm{r}=-0,41$ & $\mathrm{r}=0,05$ \\
\hline & $\mathrm{P}=0,34$ & $\mathrm{P}=0,18$ & $\mathrm{P}=0,87$ \\
\hline \multirow[t]{2}{*}{ Milho } & $r=0,37$ & $\mathrm{r}=-0,20$ & $\mathrm{r}=0,07$ \\
\hline & $\mathrm{P}=0,23$ & $\mathrm{P}=0,54$ & $\mathrm{P}=0,83$ \\
\hline \multirow[t]{2}{*}{ Soja } & $\mathrm{ND}^{*}$ & $\mathrm{r}=0,18$ & $\mathrm{r}=0,02$ \\
\hline & & $P=0,58$ & $P=0,96$ \\
\hline
\end{tabular}

*ND - Não disponível FONTE:INMET/IBGE (2020)
De acordo com Bernarde (2014) e Nogueira et al. (2016) as serpentes sofrem influência das condições ambientais. Reduzem sua taxa metabólica quando são expostas às condições desfavoráveis do ambiente, como temperaturas maiores que $29^{\circ} \mathrm{C}$ ou menores que $24^{\circ} \mathrm{C}$. Portanto, elas restringem suas atividades concominante com a ausência de chuvas, quando também ocorre a diminuição de presas disponíveis para se alimentarem.

No Tocantins, os meses de agosto e setembro (o auge da estiagem) registram as maiores temperaturas do ano, que podem ultrapassar os $40^{\circ} \mathrm{C}$, com os menores indíces pluviométricos e umidade relativa do ar. Estas características ambientais limitam o deslocamento das serpentes, que irão se manter em locais que lhes propiciem melhor conforto térmico, o que reduz a possibilidade de encontro com os humanos e consequentemente, os acidentes. 
Outra questão importante é o período reprodutivo desses animais, que também apresenta uma relação com a sazonalidade. As serpentes se reproduzem entre os meses de abril a junho, nessa fase se alimentam preferencialmente de roedores (início da estiagem). Os filhotes nascem quando iniciam as chuvas, em novembro, o período coincide com a explosão populacional de anfíbios, animais que servirão de alimento para os filhotes das serpentes nos seus primeiros meses de vida (Almeida-Santos e Orsi, 2002; Bernarde, 2014). Portanto, no primeiro trimestre do ano é de se esperar o aumento do encontro das serpentes com seres humanos, principalmente dos filhotes.

Outros trabalhos trazem a sazonalidade como fator determinante para os acidentes ofídicos, especialmente com a estação das chuvas, à umidade relativa do ar e à temperatura do ar, em diferentes regiões do país, como os estudos de Pinho et al. (2008), Bernarde et al. (2015), e também no Tocantins, por Leobas et al. (2016).

Apesar de não ter sido observada a relação do acidente botrópico com o avanço agrícola, é importante destacar que a população rural continua representando grupo de risco para o acidente ofídico. No período chuvoso, ocorre aumento da atividade humana nas áreas rurais devido à atividade agrícola, com o plantio no início das chuvas, nos meses de outubro a novembro, e colheita entre os meses de janeiro ao início de junho, meses de maior registro de acidentes ofídicos. Esse é um fator importante a ser considerado nos municípios, uma vez que, a época do ano com maior probabilidade de encontro com as serpentes coincide com o período de plantio.

Portanto, a biologia das serpentes, as questões climáticas e a atividade agrícola são características importantes que devem ser consideradas no acidente botrópico, contudo, a variabilidade de cada região precisa ser avaliada. Por isso, são necessários estudos que possibilitem analisar esses fatores, a fim de, determinar melhor o que influencia o acidente botrópico, para reduzir o impacto gerado com o acidente, no homem e nas serpentes.

\section{CONCLUSÃO}

Os resultados epidemiológicos apontam que a faixa da população em atividade laboral ativa, atingindo trabalhadores do sexo masculino é mais exposta aos acidentes botrópicos, com acidentes que manifestam alterações clínicas leves a moderadas, mas que podem levar à óbito.

Os fatores ambientais correlacionados com os acidentes botrópicos foram os climáticos (temperatura do ar, umidade relativa do ar e pluviometria) e a relação dos acidentes com as atividades rurais precisam de mais investigação.

A relação dessas variáveis climáticas com o acidente botrópico pode ser utilizada para elaborar medidas de profilaxia nas diferentes regiões do Estado. 69 Portanto, recomenda-se cuidado redobrado no período de chuva, principalmente para os municípios de maior registro dos acidentes, e para prevenir o contato direto com as serpentes, o uso de equipamentos de proteção (botas e luvas) é essencial.

\section{AGRADECIMENTO}

Os autores agradecem à Universidade Federal do Tocantins e ao Estado do Tocantins. À CAPES (Edital PROCAD 2013) e a Fundação de Amparo à Pesquisa do Estado do Tocantins/FAPT (Edital PPSUS 01/2018, Convênio 835798/2016), pelo apoio financeiro e bolsas concedidas.

Todos os autores declararam não haver qualquer potencial conflito de interesses referente a este artigo. 


\section{REFERÊNCIAS}

ALCÂNTARA, J. A., BERNARDE, P. S., SACHETT, J., DA SILVA, A. M., VALENTE, S. F., PEIXOTO, H. M., LACERDA, M., OLIVEIRA, M. R., SARAIVA, I., SAMPAIO, V. S., \& MONTEIRO, W. M. Stepping into a dangerous quagmire: Macroecological determinants of Bothrops envenomings, Brazilian Amazon. PloS one, v. 13, n. $12,2018$.

ALMEIDA-SANTOS, S.M.; ORSI, A.M. Ciclo reprodutivo de Crotalus durissus e Bothrops jararaca (Serpentes Viperidae): morfologia e função dos ovidutos. Rev. Bras. Reprod. Anim. v. 26, pg.109-112. São Paulo, 2002

BERNARDE, P. S.; MOTA-DA-SILVA, A.; ABREU, L. C. Ofidismo no estado do Acre - Brasil. Journal of Amazon Health Science, Cruzeiro do Sul - Acre, v. 1, n. 2, 2015.

BERNARDE, P. S. Serpentes peçonhentas e acidentes ofídicos no Brasil. São Paulo: Anolis Books, 2014.

BOCHNER, R.; STRUCHINER, C.J. Epidemiologia dos acidentes ofídicos nos últimos 100 anos no Brasil: uma revisão. Cadernos de Saúde Pública, v. 19, n. 1, p. 7-16, 2003.

BRASIL. MINISTÉRIO DO MEIO AMBIENTE. Plano de ação para prevenção e controle do desmatamento e das queimadas: cerrado. Brasília: MMA, 2011.

BRIDGEWATER, S.; RATTER, J.A.; RIBEIRO, J. F. Biogeographic patterns, $\beta$-diversity and dominance in the cerrado biome of Brazil. Biodiversity and Conservation, Kluwer Academic Publishers, v. 13, p. 2295-2318, 2004.

CAVALCANTI, R.B.; CARDINOT, G.; CEOTTO, P.; PINHEIRO, R.S. Biomas brasileiros: retratos de um país plural. Rio de Janeiro: Casa da Palavra Produção Editorial e Conservação Internacional. p.57-91, 2012.

INMET, Instituto Nacional de Meteorologia. BDMEP - Dados históricos (www.inmet.gov.br). 2020

INSTITUTO Brasileiro de Geografia e Estatística (IBGE). Mapa de Biomas e de Vegetação. Disponivel em: <Instituto Brasileiro de Geografia e Estatística (IBGE).>. Acesso em: 03 abril 2020.

LIMA, A.C.S.F.; CAMPOS, C.E.C.; RIBEIRO, J.R. Perfil epidemiológico de acidentes ofídicos do Estado do Amapá. Revista da Sociedade Brasileira de Medicina Tropical, v. 42, n. 3, p. 329-335, 2009.

LIMA, J. S. Perfil dos acidentes ofídicos no norte do Estado de Minas Gerais, Brasil. Revista da Sociedade Brasileira de Medicina Tropical. Belo Horizonte, v, 42, n. 5, p. 561-564, 2009.

LEOBAS G. F.; FEITOSA, S. B.; SEIBERT, C. S. Acidentes por animais peçonhentos no Estado do Tocantins: aspectos clínico-epidemiológicos.

DESAFIOS: Revista Interdisciplinar da Universidade Federal do Tocantins, v. 2. n. 2, p.2 69282, 2016.

NOGUEIRA, C.; MARQUES, O.; ETEROVIC, A.; SAZIMA, I. Serpentes do cerrado. Holos editora, 2016.

NOGUEIRA, C.C.; ARGÔLO J.S.A.; ARZAMENDIA V.; AZEVEDO A.J.; BARBO E.F.; BÉRNILS S. R.; BOLOCHIO E.B.; BORGESMARTINS M.; BRASIL-GODINHO M.; BRAZ H.; BUONONATO A.M.; CISNEROS-HEREDIA D. F.; COLLI G.R.; COSTA H.C.; FRANCO F.L.; GIRAUDO A.; RODRIGO C. GONZALEZ R.G.; GUEDES T.; HOOGMOED M.S.; MARQUES O.A.V.; MONTINGELLI G.G.; PASSOS P.; PRUDENTE A.L.C.; RIVAS G.A.; PAOLA M. 70 SANCHEZ P.M.; SERRANO F.C.; JÚNIOR N.J.S.; STRÜSSMANN C.; VIEIRA-ALENCAR J.P.S.; ZAHER H.; SAWAYA R.J.; MARTINS M. Atlas of Brazilian Snakes: Verified Point-Locality Maps to Mitigate the Wallacean Shortfall in a Megadiverse Snake Fauna. South American Journal of Herpetology, v. 14, p. 1-274, 2019.

PARISE, E. V. Vigilância e monitoramento dos acidentes por animais peçonhentos no município de Palmas, Tocantins, Brasil. HYGEIA - Revista Brasileira de Geografia Médica e da Saúde, v. 12, n. 22, p. 72-87, 2016.

PAULA, R. C. M. F. Perfil epidemiológicos dos casos de acidentes ofídicos atendidos no Hospital de Doenças Tropicais de Araguaína-TO (Triênio 2007-2009). São Paulo: Universidade de São Paulo, 104p. Dissertação (mestrado), Universidade de São Paulo, São Paulo. 2010.

PINHO, F. M. O.; YU, L.; BURDMANN, E. A. Snakebite-induced acute kidney injury in Latin America. Semin Nephrol, v. 28, n. 4, p. 354-62, 2008.

SANTANA, V. T. P.; SUCHARA, E. A. Epidemiologia dos acidentes com animais peçonhentos registrados em Nova Xavantina - MT. Revista de 
Epidemiologia e Controle de Infecção, v. 5, p. 141146, 2015.

SANTOS, A. L.; FEITOSA, S. B.; SANO-MARTINS, I. S.; MORENA, D. D. S; SEIBERT, C. S. Estudo retrospectivo dos acidentes por serpentes atendidos no Hospital Geral de Palmas -TO, no período de 2010 e 2011. Desafios: Revista Interdisciplinar da Universidade Federal do Tocantins -, Palmas, v. 1, n. 1, p. 226-244, 2014.

SEAGRO. Agricultura, 2018. Disponivel em: <https://seagro.to.gov.br/agricultura/>.
SILVA, R. C. C. O ambiente e a diversidade das serpentes no estado do Tocantins - Brasil Palmas - TO. 155 f. Dissertação (Mestrado) - Curso de Ciências do Ambiente, Programa de Pós-graduação, Universidade Federal do Tocantins, Palmas, 2017.

SINAN/SVS/MS. Sistema de Informação de Agravos de Notificação-SINAN, Ministério da Saúde, 2020.

XIMENES, L. M. A. D. S.; SANTOS, L.; SANTOS, M. Perfil dos acidentes ofídicos ocorridos no município de Alta Floresta - Mato Grosso. Enciclopédia Biosfera, Centro Científico Conhecer- Goiânia,v. 15, p. 1458, 2018. 